\title{
A Semi-Structured Approach to Sketch Mapping and Qualitative GIS
}

Corresponding Author:

Christopher G. Prener, Ph.D.

Assistant Professor of Sociology

Saint Louis University

chris.prener@slu.edu

ORCID - 0000-0002-4310-9888

Current Mailing Address for the Corresponding Author:

Department of Sociology and Anthropology

1918 Morrissey Hall

Saint Louis University

St. Louis, MO 63108

Keywords:

qualitative GIS, sketch mapping, crime, cities, open source software

\section{Acknowledgements:}

The author wishes to thank Ava Gagner for her assistance in developing the map instrument and for providing help with the data collection process.

This manuscript is currently under peer review. 


\section{A Semi-Structured Approach to Sketch Mapping and Qualitative GIS}

Sketch mapping provides an intuitive means for collecting respondents' impressions about space and place alongside qualitative data collection approaches. However, the data collected are often challenging to work with, particularly with digitization and visualization. After reviewing these challenges, this article introduces a "semi-structured" approach to sketch map data collection, digitization, and analysis as well as an open source software package, "qualmap," that implements tools for digitizing these data in the programming language $\mathrm{R}$. This approach retains the cartographically accurate base maps used in other sketch map research. However, it superimposes an areal unit of the researchers' choice to aid in digitization and analysis. This use of a standard areal unit opens up additional cartographic and analytic possibilities with sketch map data. After describing this approach and the accompanying software, this article illustrates its applicability with a sample project that analyzes perceptions of space collected from a convenience sample of college students. The resulting maps' clustering identifies the presence of a "bubble" around their campus that captures much of the travel activity students engage in every week. These data are paired with crime data, which illustrates possible mixed methods extensions of this "semi-structured" approach to sketch mapping. 


\section{A Semi-Structured Approach to Sketch Mapping and Qualitative GIS}

Qualitative techniques that utilize spatial data can provide an engaging, illuminating, and immersive experience both for data collection and communicating findings. Within geography, the predominant approach to integrating qualitative and spatial data has been through techniques known alternatively as sketch, mental, cognitive, and perceptual mapping (Boschmann and Cubbon 2014; Brennan-Horley and Gibson 2009; Tuan 1975). Sketch mapping, the technique utilized in this paper, uses a pre-existing, cartographically accurate base map to guide spatial data collection where the goal is to understand the distribution of activities or perceptions across space (Boschmann and Cubbon 2014). Rather than being the sole form of data collected, sketch mapping is typically a supplementary activity alongside focus group (e.g. Fielding and Cisneros-Puebla 2010) or interview data (e.g. Brennan-Horley and Gibson 2009). The digitization of these data and subsequent geographic information systems ("GIS") analysis of them is an integral part of the analytic process, but one that is difficult to complete. The very features of sketch map data that make them analytically powerful, the way that they reveal perceptual patterns across space, also "makes collating responses with a GIS problematic, if not impossible" (Brennan-Horley and Gibson 2009, 2604). Digitizing "sketch map" data is often limited to scanning it and providing examples of participants' maps while some researchers provide no visual summaries of their data at all. When composite maps of all respondents are included, a 
multitude of different approaches are utilized to demonstrate where perceptions or activities cluster in a particular place.

This article introduces a novel approach for working with "sketch map" data that I term "semi-structured sketch mapping." Heavily influenced by Brennan-Horley and Gibson's (2009) work, this approach is rooted firmly in the tradition of sketch mapping, but also provides an opinionated framework for digitizing participants' map data in ways that allow for extensible and extensive analysis. After reviewing the tradition of qualitative GIS and sketch mapping specifically, this article introduces the semistructured approach. The term "semi-structured" is used to characterized the use of standarized areal units for digitizing participants' qualitative data generated through sketch mapping. This approach is illustrated with a project that investigates the presence of a "bubble" around a U.S. university campus. By pairing students' sketch map data, which illustrate this "bubble," with crime data, this sample project highlights the value of semi-structured sketch mapping for both assessing the spatial extent of qualitative phenomena and for pairing qualitative data with data from other sources.

\section{Background}

Geographic Information Systems (or "GIS"), the computer hardware and software used for processing and visualizing spatial data, have existed since the 1960s. Over time, expansions in computational power have expanded the possibilities for working with spatial data, and the development of open source software has decreased many of the 
barriers of entry for working with these data. This continued evolution comes, however, amid long been tensions in the GIS community along a variety of axes (see Goodchild 2000), perhaps none more relevant here than debates over the positivist epistemological orientation of GIS that feminist geographers have long criticized (Elwood and Cope 2009) and questions about where GIS fits into the traditional qualitative-quantitative methodological divides (Pavlovskaya 2009).

\section{Qualitative GIS}

While critiques of positivism within the social sciences and humanities generally (see Agger 1991 for an overview) and in geography specifically (e.g., Smith 1979) developed temporally alongside modern GIS systems (Goodchild 2000), it was not until the early 1990s that geographers began to frame GIS itself as a positivist approach (Elwood and Cope 2009; Lake 1993; Pickles 1995). During this period, feminist geographers and GIS practitioners began to sharply critique this positivist orientation (Elwood and Cope 2009; Pavlovskaya 2006, 2009; Kwan 2002) and argue for alternative means of capturing individuals' lived experiences of space and place.

These critiques by qualitative geographers (Bell and Reed 2006; Kwan 2002;

Kwan and Knigge 2006; Matthews, Detwiler, and Burton 2006; Pavlovskaya 2002, 2009) led over time to the development of "qualitative GIS" as an overarching term for both qualitative and mixed methods approaches to spatial data collection and analyses (Elwood and Cope 2009). What ties these varying approaches together is an 
epistemologically distinct view of spatial data that differs from numerical approaches to GIS. Most importantly, for the present context, these critiques have included the "absolute" way in which GIS conceptualizes space, whereas individuals construct their own understandings of relative flows, places, and networks (Pavlovskaya 2009).

The use of qualitative GIS to understand these phenomena has sometimes been couched by researchers in terms of "participatory mapping" (Hohenthal, Minoia, and Pellikka 2017). Including indigenous groups or other often marginalized communities in the co-production of maps that involve their neighborhoods or land not only inverts this power relationship but also can create alternative ways of understanding how space and place intersect in peoples' lived experiences, cultures, and ways of being.

In order to capture these alternative views of space and place, geographers have experimented with spatial data using traditionally "qualitative" approaches like interviewing, focus groups, and ethnography. The specific techniques used to capture spatial knowledge during these qualitative encounters have their roots in classic works like Kevin Lynch's (1960) conception of "mental maps." In their purest form, these maps are drawn on a blank piece of paper. The greatest strength of mental mapping lies in these unstructured but detailed representations of geography, but this strength is also the signature weakness of the method. The lack of grounding in a known coordinate system is a property that, as Brennan-Horley and Gibson (2009) note, contributes to the immense difficulty of working with "mental map" data. 


\section{Sketch Mapping}

In response to these barriers, some researchers recently have advocated for a distinct approach that on a cartographically accurate base map that can be drawn upon and annotated (Matei, Ball-Rokeach, and Qiu 2001; Brennan-Horley and Gibson 2009; Boschmann and Cubbon 2014). Known generally as "sketch mapping" (Boschmann and Cubbon 2014), ${ }^{1}$ this is an attempt to ease the analyses of these data by relying on a known projected coordinate system to facilitate the digitization process.

As Table 1 indicates, geographers and others have employed sketch mapping in a variety of contexts. These substantive areas include documenting community fears of crime (e.g. Matei, Ball-Rokeach, and Qiu 2001; Curtis et al. 2014, 2019), the concentration of "creative industry" (e.g. Bereitschaft 2018; Brennan-Horley and Gibson 2009; Brennan-Horley et al. 2010), access to jobs (e.g. Boschmann 2011), residents' physical activity (e.g. Manton et al. 2016; Styers et al. 2018; Wridt 2010), land and water use (e.g. De Oliveira D'Antona, Cak, and VanWey 2008; Hall-Arber and St Martin 2007; Hohenthal, Minoia, and Pellikka 2017; Weiner and Harris 2003), public health (e.g. Dongus et al. 2007), and environmental hazards (e.g. Coquet, Mercier, and Fleury-Bahi 2018; Klonner et al. 2018; O’Neill et al. 2015; Sletto et al. 2010).

Researchers have also used them widely with both individual respondents and focus groups. Their use in focus groups points to multiple alternative goals for sketch mapping. Curtis (2016) notes that mental mapping and sketch mapping together have been used to explore "behavior," "knowledge," "perception," and "preference." Focus 
groups, in particular, are often used to develop consensus views of knowledge from participants.

\section{$<<<$ TABLE 1 ABOUT HERE $>>>>$}

Another point of variation is what researchers do with their sketch map data. As Table 1 indicates, not all researchers even include maps in their articles. Those that do include maps are divided between showing only a selection of individual maps (e.g., De Oliveira D'Antona, Cak, and VanWey 2008; Dongus et al. 2007; Fielding and CisnerosPuebla 2010; Weiner and Harris 2003). Others have pursued a variety of means for creating composites of multiple respondents' maps (e.g., Boschmann 2011; BrennanHorley and Gibson 2009; Manton et al. 2016; Wridt 2010). What is particularly striking, however, is that there is little overlap in the approaches researchers have followed in generating these maps. Most researchers appear to forge independent paths for determining how best to digitize, visualize, and (for some) further analyze their respondents' data.

Indeed, there are few articles specifically about the methods themselves for creating composite maps from multiple respondents (there are three notable exceptions Brennan-Horley and Gibson 2009; Brennan-Horley et al. 2010; Sloan et al. 2016). One approach has been to convert individuals' maps into rasters using the same projection used to create the base maps (Brennan-Horley and Gibson 2009; Brennan-Horley et al. 2010; Sloan et al. 2016). Others have digitized their respondents' annotations as vector data and identified areas of overlap using points or lines. There is little discussion, 
however, about best practices or tradeoffs for pursuing different approaches. Moreover, papers identify areas of overlap between respondents without going further, such as applying spatial statistics to respondents' data (see Matei, Ball-Rokeach, and Qiu 2001; O'Neill et al. 2015; Curtis et al. 2019 for notable departures) or integrating these data with data from other sources (Bereitschaft 2018). While there is increasing interest in capturing perceptions of space (Curtis 2016) on cartographically accurate base maps (Boschmann and Cubbon 2014), there remains much to be done in terms of clarifying approaches to integrate this into research designs.

\section{Towards a Mixed Methods GIS}

Given the epistemological roots of qualitative GIS more generally, and the undercurrent of critique present in the participatory GIS literature, using spatial statistics or integrating sketch map data with other sources may be somewhat controversial. Much of the early focus of qualitative GIS was on differentiating itself from "numerical" or "quantitate" approaches to GIS (see Pavlovskaya 2006), traits shared by the embrace of participatory GIS. As in other fields (see, for example, Dohan and Sánchez-Jankowski 1998), here have been debates for decades now about the place of computational techniques in qualitative research. As (Pavlovskaya 2009) notes, computerization is not the same as quantification. The suggestion that folding qualitative data into statistical analyses is a possibility should not be seen as an effort to undermine the vital legacy of post-positivist research methods in geography and GIS. 
Instead, with that context in mind, I want to suggest that there are a multiplicity of ways in which qualitative GIS data can inform research insights and contribute to a variety of research designs.

The multiplicity of ways in which qualitative GIS can support research is a natural segue into a final tension for the field, which is how we think about the role of qualitative GIS in a research world where academics often rely on bifurcated qualitative and qualitative labels. (Pavlovskaya 2009) argues that most GIS applications are qualitative rather than quantitative. Designs that leverage both qualitative data collection on base maps as well as spatial statistics (Matei, Ball-Rokeach, and Qiu 2001; O'Neill et al. 2015; Curtis et al. 2019) or compare qualitative distributions to other data sources (e.g., Bereitschaft 2018) suggest that distinguishing GIS as either a quantitative or qualitative approach may miss the fundamental ways in which GIS mixes methods from a variety of approaches.

\section{A Semi-Structured Approach to GIS}

The semi-structured approach to qualitative GIS shared here is a platform conceived of as an inherently mixed methods approach to "sketch mapping." Rather than relying on sharing one or two example maps, or rasterizing participants' data, the semi-structured methodology presented here relies on the alignment of data with areal units at all stages of data collection and analysis. This alignment with areal units is why I characterize the method as "semi-structured." Much as in a semi-structured interview, 
there is license to explore space and place in however the participant chooses to do so, but there is a structure that shapes the data collection and analysis process. The approach laid out here is an "opinionated" one, a term borrowed from software development (Eccles 2015) that is finding increased use in the data science world as well (Parker 2017). In both its original context and its use in data science, the idea of an "opinionated" programming language or analysis framework is that it encourages the end-user to follow a particular set of principles.

To that end, the process described here as well as the accompanying software, makes a series of assumptions about how data are being collected and digitized.

Importantly, this does not preclude or dismiss other approaches to digitized qualitative GIS data, nor does it assume that the semi-structured approach to qualitative GIS is inherently more valid than any of the prior approaches to working with these types of data. Instead, the term "opinionated" captures the internal logic of the method presented here. It also describes the software development paradigm that the accompanying software, qualmap $\left(<\right.$ citation redacted $>$ ), ${ }^{2}$ reflects (Parker 2017). The semi-structured GIS workflow (see Figure 1) captures this process, and the following sections walk through each phase of this "opinionated" process.

\section{$<<<<$ FIGURE 1 ABOUT HERE $>>>>$}

Selecting an Areal Unit 
An initial task for many researchers working with spatial data is to select an appropriate unit of analysis, and this semi-structured approach to qualitative GIS is no different. The areal units used can be pre-existing constructs, such as census enumeration units (like the American census blocks, block groups, or tracts). Alternatively, researchers can use any form and size of tessellation (Gold 2016), including square grids, triangles, or hexagons. Researchers could similarly select predefined administrative units like neighborhoods, political precincts, or even counties as areal units. The variety of options gives researchers maximal opportunity to select areal units that are appropriate for their research questions. These areal units will be used for digitization, and allow for a summary of how frequently participants mention particular areas, providing a clear means for analyzing and communicating findings from studies that utilize semi-structured sketch mapping.

\section{Base Map Preparation}

Like standard approaches to sketch mapping, this semi-structured approach utilizes large format base maps given to participants during interviews or focus groups. Researchers are encouraged to follow established best practices for producing these, such as providing important contextual information (such as bodies of water and other significant landmarks that help participants "navigate" the sketch map). Landmarks are likewise useful for helping participants identify the optimal locations for applying marks to the map. These locations should not be prejudicial, but should instead be 
thoughtfully selected to help participants, particularly those who do not view themselves as strong map readers, complete the exercise with minimal exertion. The risk of prejudicial landmarks is that they may coax respondents to identify particular places more frequently than they would if the particular landmark was not labeled. The base map itself should have the selected areal units overlaid on the other layers to facilitate efficient digitization. For the easiest digitization, providing identification numbers for each areal unit is also recommended (see Figure 2).

\section{$<<<<$ FIGURE 2 ABOUT HERE $>>>>$}

\section{Data Collection}

Large format printing, such as eleven inch by seventeen inch sheets, enables large scale mapping that is easy to navigate for participants. The design of these map layout again parallels the techniques documented in prior studies of sketch map work. These map sheets should include not only the base map but also the relevant prompt. These sheets can also include standard map layout features, such as scale bars and legends, and also may include a sample of what participant markings should look like (typically circles drawn around relevant portions of the map, termed "clusters" here). During the data collection process, the interviewer should introduce the map, give participants an overview of how to navigate it and what participant markings should entail, and then describe the relevant prompt or prompts that the map is to be used for by participants for annotation. 
One decision for researchers to make is whether or not they want to collect multiple prompts worth of data on a single sketch map. Using a single map could potentially reduce confusion for respondents about which map sheet is for which prompt, reduce clutter for researchers, and minimize printing costs. However, a single base map requires a second-level of detail because participants must somehow differentiate between prompts or constructs by alternating the color used to mark-up the map, labeling their marks, or using different symbols. In contrast, limiting each map sheet to a single construct reduces the burden placed on respondents to differentiate the way that they record clusters on the map. Regardless of which approach is taken, researchers are strongly encouraged to pilot their maps before beginning formal data collection.

\section{Manual Verification}

In-between the process of collecting data and the steps required for digitization, there is a recommended process of marking each map layout. Steps include adding a participation or respondent identification number to each map layout as well as numbering each cluster the participant drew. This second step connects the physical map layouts to the code and digital versions of the data. In particular, numbering each cluster makes the code more expressive by clearly linking it to a physical entity. Numbering also makes the process of auditing and replicating a study easier, since there is a concrete link between the physical maps, the code used to digitize them, and the 
resulting data object. An example of this manual verification work can be seen in the numbering applied to each cluster visible in Figure 2.

\section{Digitization}

In a novel departure from other forms of sketch mapping, this semi-structured data collection process has a companion digitization process that is highlighted in Figure 1. A software package named qualmap, written the open-source programming language R (R Core Team 2019), allows for efficient data entry, validation, and summary. Like other R packages, particularly in the tidyverse (Wickham et al. 2019), qualmap is oriented around functions that represent particular verbs. qualmap's verbs are summarized in Table 1, and each is named to evoke the key behavior each function encompasses.

\section{$<<<$ TABLE 2 ABOUT HERE $>>>>$}

Each cluster, i.e. a participant marking, is entered individually using the identification numbers of the areal unit that this cluster either (a) touches or (b) includes the centroid. These digitized clusters are validated to ensure they each match valid identification numbers. Clusters can also be easily projected and, therefore, visually inspected using an interactive map created automatically with the leaflet (Cheng, Karambelkar, and Xie 2019) package for R. Part of the cluster creation process also asks end-users to enter metadata about the respondent as well as the analytic category the cluster represents, which serves as a way to map and analyze different 
qualitative constructs. Clusters will always contain a row for each areal unit included in the cluster. A cluster that encompasses three grid squares, for example, will be represented by a data object that contains three rows.

Once the end-user has entered multiple clusters, the next step is to combine them into a single object. These are "long" data in the sense that they contain multiple clusters for one or more participants, and the combined output may contain multiple rows for the same feature if it has been included in multiple clusters by the respondent. Manipulating sketch map data this way is particularly useful for storing an entire study's data in a single data table and exporting it for on-disk storage. Moreover, because it contains references to the individual features, it can be stored as a lightweight text file (e.g. a .csv) rather than a potentially large vector or raster file (e.g. a "shapefile" or image file). If participants' data are stored on-disk in separate files, which may be preferable in large projects, the "bind" verb can be used to combine them all into a single, project-wide data table once they are re-imported into $\mathrm{R}$.

However they are stored (as a single output file or as many) prior to analysis, these "long" data present a challenge for cartographically representing the cluster data on a map. The final verb, "summarize," summarizes clusters for a particular category, generating a count of clusters that include each areal unit in the validation data. The summarization process results in an object with the same number of observations as the original shapefile. If there were twenty grid squares in a hypothetical data set, the object would have a row for each grid square. This final object is an sf object (Pebesma 2018), 
meaning it ready-made for mapping, geoprocessing, or exporting as a shapefile or other geographic data format.

\section{Cartographic and Analytic Possibilities}

Once a researcher has summarized their participants' clusters, a range of analytic possibilities are open to them. The summarization process produces counts within areal units, which lend themselves to thematic choropleth mapping as well as the production of bivariate plots (or maps) that compare the count of clusters to another variable within the scope of the given research project. Likewise, these data can be easily used in a-spatial statistical analyses, analyzed for spatial autocorrelation, and used in spatial modeling.

$\mathrm{R}$ provides an excellent platform for conducting both mapping and these statistical analyses, and the data structures that qualmap creates can be easily mapped as sf objects and converted to sp objects (Bivand, Pebesma, and Gomez-Rubio 2013; Pebesma and Bivand 2005) for spatial statistical analyses. There are also dedicated packages in $\mathrm{R}$ for quickly downloading demographic data for a variety of countries. In the U.S., the tidycensus (K. Walker 2019a) and tigris (K. Walker 2019b) packages provide seamless access to the U.S. Census Bureau's API. These two packages provide easy access to population counts, demographic data, and other characteristics about census geographies along with the the geometric data needed to map these various geographies. There are also a number of options for mapping within the $\mathrm{R}$ ecosystem, 
including ggplot2 (Wickham 2016) and tmap (Tennekes 2018). Moreover, RStudio's specific integrations for working between $\mathrm{R}$ and Python mean that qualmap centric workflows can swiftly produce data that can be analyzed with pysal (Rey and Anselin 2010) or other Python libraries as well.

This range of options can augment researchers' qualitative data. The semistructured approach fits into a mixed-methods framework for understanding both the qualitative impressions of space and place that qualitative GIS excels at producing. At the same time, it also allows researchers to understand how qualitative impressions reflect broader patterns within cities and other areas where researchers may use this technique for data collection. This semi-structured process, therefore, leverages the "best of both worlds." It allows researchers to quickly draw conclusions from qualitative data by paring the data with interviews or focus group transcripts, visualizing participants' clusters with maps and other approaches, and using qualitative data as outcomes or independent variables in statistical models.

\section{A Sample Project}

Implementing these techniques in a project requires several decisions and analytic steps, best illustrated by providing a detailed description of a sample project. This section walks through that process, introducing a toy data set that accompanies both this manuscript and the qualmap software. This data set was created in the spring of 2019, specifically to demonstrate the semi-structured GIS workflow. Though it is a pilot 
project whose goals had to do with developing the software, the questions themselves originated in more substantial interest in students' exposure to and engagement with neighborhoods adjacent to their campus. Termed the "bubble" by students at Saint Louis University, the sphere of the campus is both physically and symbolically bounded. This understanding of college campuses' spatiality and students' relationship to it corresponds with a small literature on the topic (see $\mathrm{Yu}$ et al. 2018). It also connects to broader literatures on placemaking and how students see themselves existing in a broader urban context (Hopkins 2011; Andersson, Sadgrove, and Valentine 2012; Holton and Riley 2013; Holton 2015, 2016; Yu et al. 2018).

For Saint Louis University in particular, this bubble takes on concerns not just about place-making but rather students' perceptions of crime and saftey in St. Louis (Wachnik 2019). Violent crime rates in St. Louis are among the highest in the nation for large cities (see Prener 2020; Sandoval 2013; T. A. Smith and Sandoval 2019a, 2019b), and so students' understandings of space may unsurprisingly be connected to perceived patterns in crime as well as whether spaces "feel" safe or not. In a recent student newspaper article on the subject, a student told the reporter that "I haven't seen a lot of St. Louis for myself, but I know that it's pretty dangerous and that there are a lot of crimes around the city." This sample project, therefore, illustrates how sketch map data can be analyzed in concert with other data such as crime rates, to "triangulate" an understanding of the campus bubble. The addition of crime data also connects this pilot project to the broader sketch mapping literature, where understanding perceptions of 
crime and fear within cities has been an active area of interest (Ceccato and Snickars

2000; Matei, Ball-Rokeach, and Qiu 2001; Doran and Lees 2005; Pain et al. 2006; Lopez and Lukinbeal 2010; Doran and Burgess 2011; Curtis et al. 2014, 2019; Sloan et al. 2016). The project also illustrates the ways in which sketch map data can be combined with other data sources to deepen mixed methods analyses (see also Bereitschaft 2018).

\section{Data and Methods}

I worked with a student researcher who helped with the initial design and data collection tasks, including the developing and piloting of a base map for the neighborhoods surrounding Saint Louis University. The full base map contained building footprints, the street grid, subway and rail lines, parks, and significant landmarks. The campus itself was defined with a hashed background to help students unfamiliar with surrounding neighborhoods root themselves to campus. Each map layout corresponded to one of the two-prompts in order to reduce the burden on participants when responding to our questions:

1. "On the map, draw circles around the areas near and on-campus that you frequently visit (at least twice per week)."

2. "On the map, draw circles around the areas near and on-campus that you regularly visit (at most three to four times per month)" 
Both map layouts included instructions to respondents that: "Your circles do not have to conform to any particular shape or size. You do not have to try and circle individual buildings." Moreover, each map layout included an example inset map showing several sample clusters. The map also included a legend and a scale bar as well. Finally, we overlaid a grid that corresponded to our areal units on top of the other base map layers. The grid was colored as a ground layer not to distract respondents, as were the labels "B-1," "B-2," and so-on. We provided these labels to make digitizing the clusters as easy as possible.

Respondents were handed packets at the beginning of the data collection sessions, which included three geography and sociology classes at Saint Louis University. A cover sheet included recruitment language approved by the Saint Louis University Institutional Review Board was paper clipped to both maps. ${ }^{3}$ Each participant received a packet, and the author or the student researcher orally introduced the study as well as each map layout and their associated prompts. Once data were collected, each cluster was digitized using $\mathrm{R}$ and RStudio. All data were digitized using the qualmap package $(<$ citation reducated $>)$ and processed into maps using tmap (Tennekes 2018). ${ }^{4}$ Additional spatial statistics, particularly Moran's I, were calculated using the sp (Pebesma and Bivand 2005; Bivand, Pebesma, and Gomez-Rubio 2013) and spdep (Bivand, Pebesma, and Gomez-Rubio 2013; Bivand and Wong 2018) packages for R. Rook spatial weights were used since the data were in a grid shape, and the $p$ values were simulated using a Monte-Carlo process with 999 simulations. 
To identify possible relationships between crime and student activity spaces, crime data for the City of St. Louis were added to the final analytical data set. These data were downloaded and validated using the compstatr package (Prener and Foeller 2019), which provides access to St. Louis Metropolitan Police Department crime data. Part 1 crimes $^{5}$ that occurred in the year before sketch map data collection (May 1st, 2018 to April 30th, 2019) were joined to grid squares to produce counts per square. The total population, using the 2018 American Community Survey (ACS) five-year estimates for census tracts, was estimated for each grid square using areal weighted interpolation. The areal package (Prener and Revord 2019) was used to produce the interpolated estimates from tract-level ACS data, and these estimates were combined with the counts of crimes to calculate crime rates per 1,000 estimated residents. These rates were then compared with the respondent's cluster data for analysis. These comparisons included the calculation of correlations using the stats package (R Core Team 2019) and the production of scatterplots using the ggplot2 (Wickham 2016) and cowplot (Wilke 2019).

Results

The map documenting the first prompt, where students frequently visit (see Figure 3), shows clustering around North Campus. We can quantify this clustering by measuring the spatial autocorrelation in these data with Moran's I. For the frequency measure, which identifies the percentage of students who visit multiple times per week, the Moran's $I$ value is 0.515 (simulated $p=0.001$ ). This Moran's $I$ indicates moderate 
clustering in these data. Both the map and the Moran's I statistic provide some evidence that a "bubble" exists around campus for the students who participated in pilot data collection, with particular emphasis placed on the North Campus area. These findings suggest that the most common areas of student activity are on and immediately adjacent to Saint Louis University's North Campus, followed by the area on and around the South Campus.

\section{$<<<<$ FIGURE 3 ABOUT HERE $>>>>$}

The second map, which shows percentage of students who visit regularly (i.e. a few times a month; see Figure 4), also shows clustering $(I=0.536$, simulated $p=$ 0.001). However, there are two notable differences. There is a higher percentage of students reporting visits to the Central West End and Forest Park Southeast neighborhoods, both of which are to the west of campus. Each offers bars and restaurants, which are concentrated in several commercial clusters. These clusters correspond to many of the individual clusters. Another notable area in the Central West End is a shopping plaza just west of campus where a grocery store is located. This map is slightly more diffuse, in that students activity spaces are slightly less concentrated around campus and spread out into some areas that surround campus to a greater degree than their frequent activities. 
These two maps, taken together, suggest that there are "activity spaces" that students frequently and regularly visit. These spaces form the core of the so-called "SLU bubble" around Saint Louis University's campus - the highest frequency areas are all immediately on or adjacent to SLU's campus. This clustering emerges visually from the choropleth maps. The degree of autocorrelation present in the respondent data are also suggestive of clustering in this bubble.

One potential explanation for this clustering, students' desire for safety in a city that has some of the highest violent crime rates in the United States, can be partially analyzed by comparing the crime rates of grid squares to these cluster data. As Figure 5 shows, these relationships are exceptionally weak for both the frequency percentages and the regular percentages. Neither shows a substantial relationship with the estimated Part 1 crime rates for each grid square. In fact, the correlations between the frequency percentages and Part 1 crimes $(r=0.029, p=0.846)$ as well as between regular percentages and Part 1 crimes $(r=0.096, p=0.512)$ are weak and not statistically significant.

\section{$<<<<$ FIGURE 5 ABOUT HERE $>>>>$}

It is possible and even probable that the lack of a substantial relationship between "activity spaces" around campus and crime rates is due to a mismatch between students' perceived beliefs about crime and the actual incidence of crime around them. Other work has identified significant clustering of violent crime in other parts of St. 
Louis that are not near campus. However, college students, particularly those who are not familiar with the city, may not have the geographic knowledge or the detailed exposure to crime data to differentiate between the actual incidence of crime and perceived risk. An essential follow-up to this pilot data would, therefore, be to understand not just the spatial extent of student "activity spaces" but also the risk students feel regarding crime victimization in neighborhoods around campus as well. Such a follow-up could likewise be explored using semi-structured sketch mapping, with prompts similar to other studies (Ceccato and Snickars 2000; Matei, Ball-Rokeach, and

Qiu 2001; Doran and Lees 2005; Pain et al. 2006; Lopez and Lukinbeal 2010; Doran and Burgess 2011; Curtis et al. 2014, 2019; Sloan et al. 2016) that seek to understand the level of perceived fear associated with particular spaces and places.

\section{Discussion}

Semi-structured sketch mapping is envisioned here as a way to link both qualitative and quantitative methodologies. It should not be viewed, however, as a replacement for other forms of qualitative GIS, nor should it be seen by researchers as an improvement on qualitative GIS as a whole. Indeed, the goal of developing the technique and its associated software is not to supplant existing approaches. Instead, this approach provides a framework for structured sketch mapping data collection, 
digitization, and analysis in situations where such a mixed-methods approach is desirable.

While semi-structured sketch mapping provides a cohesive link between qualitative representations captured with simple tools like a pen and a sheet of paper and computationally intensive tools like spatial modeling, some researchers may not roundly welcome this linkage. Geographers working in qualitative GIS have long positioned it as epistemologically distinct from the idea that of GIS as a quantitative field (Pavlovskaya 2009). The fundamental power relations within "traditional" GIS that have been critiqued by scholars who positioned themselves as proponents of qualitative GIS remain in this approach. However, as (Pavlovskaya 2009) notes, the old paradigm of quantitative work being viewed by researchers as positivist and qualitative work as un-scientific has faded to a degree. Qualitative work is no longer the sole domain of feminist, poststructuralist, and neo-Marxist geography, and researchers increasingly view mixed methods work as a critical, valid approach to scientific research (Creswell and Creswell 2017; Knigge and Cope 2006; Teddlie and Tashakkori 2011).

Indeed, the idea of complementary research designs where quantitative work informs qualitative approaches or vice versa (Morse 2016) is at the heart of how researchers frame many contemporary mixed-methods research designs. Semi-structured sketch mapping allows this to be taken a step further by offering an interactive approach that blends both qualitative and quantitative techniques simultaneously. Researchers can, therefore, pair interview and focus group data with spatial 
representations of other data, such as the demographic or administrative data. This pairing opens the door to more advanced spatial statistics and modeling.

A full-fledged analysis for the pilot study included here could therefore integrate either interview or focus group data to understand how students themselves think about the phenomenon of the "SLU bubble." These approaches could also help identify how activities vary between campus and the less frequent visits to the neighborhoods to the west. Other opportunities for quantitative analysis could include understanding how students' patterns correspond to demographic patterns. Beyond calculating crime rates, the grid created for data collection could provide a basis for interpolating data on neighborhood race and socioeconomic status from readily available census geographies.

As I noted previously, the R ecosystem includes tools for both downloading these data (the tidycensus package, K. Walker 2019a) and interpolating them (the areal package, Prener and Revord 2019). Other data sources that may help us understand students' interactions with space, such as additional indicators of neighborhood disorder, could also be calculated for these same geographies.

\section{Limitations}

While the "semi-structured" approach to qualitative GIS and mixed methods GIS creates a well-defined structure for collecting and digitizing data, it does have some limitations. Unsurprisingly, it is not appropriate for more loosely structured "mental mapping" exercises in the vein of Lynch (1965). The nature of how clusters align with 
areal units is also a limitation, in the sense that it is not a precise rendering of the clusters. These precise scans of clusters have appeared in some sketch map research (e.g., Boschmann and Cubbon 2014). The degree to which the precision and generalization of these entities matters for researchers' questions should be the subject of future research.

However, there is reason to believe both generalized clusters and precise digitization each have a role in sketch mapping. On the one hand, participants' impressions of space and place are likely to be inexact, and thus the precision of their markings on the map should not be treated as absolute in many if not most cases. On the other hand, areal units may be significantly larger than individual clusters and thus have the effect of overstating the spatial extent of respondents' data. The selection of an appropriate areal unit is, therefore, essential. In particular, it is critical to ensure that the selected unit is not too large so as not to distort the underlying data dramatically. The impact of varying sizes of areal units should be a subject of future research.

Another limitation is the potential identifiability of participants' data.

Identifiability is not a concern that frequently appears in the sketch mapping literature, and some work (e.g. Boschmann and Cubbon 2014) shows individual maps that could potentially reveal participants' home locations and, therefore, their identity. Revealing sensitive information is undoubtedly a broader concern with spatial data (see Montjoye et al. 2013), but bears emphasis here because of the focus placed on open science and reproducibility in this article. In the sketch map articles reviewed for Table 1, several 
papers included examples of participants' data or composite maps with detailed data that could potentially identify individual respondents. This only should be done with significant attention paid to the possible ramifications of re-identification.

\section{Conclusion}

As a subset of qualitative GIS work, sketch mapping can collect spatial data from participants in ways that open up a variety of options for visualizing and further analyzing these qualitative data. Unlike mental mapping (Lynch 1960), the use of cartographically accurate base maps enables a multitude of options. However, the literature on sketch mapping (see Table 1) often invents or reinvents strategies for digitizing these data and displaying results. There are few standards or "best practices" that have been detailed by practitioners apart from some documentation of techniques for rasterizing data.

The goal of the qualmap package for $\mathrm{R}$ is to provide one possible approach to collecting, digitizing, and visualizing these sketch map data in a well-documented manner. Moreover, because it relies on open-source software already in everyday use among academics, it potentially lowers the bar for others to access to implement these techniques. Along with a well-documented workflow for implementing sketch mapping in mixed-methods research, this semi-structured approach to sketch mapping can reveal patterns in both small and large studies that include spatial components, such as the sample project that investigates the presence of a "bubble" around an urban university 
campus. Developing a variety of sketch mapping techniques for both data collection and digitization helps to expand the reach of this technique and continue to grow its presence in a variety of substantive literatures. 
Tables

Table 1. Overview of the "Sketch Mapping" Literature

\begin{tabular}{|c|c|c|c|c|}
\hline Year & Authors & Domain & Unit & $\begin{array}{l}\text { Composite } \\
\text { Maps }\end{array}$ \\
\hline 2000 & Ceccato and Snickars & crime & individual & yes \\
\hline \multirow[t]{2}{*}{2001} & Coulton et al. & urban space & individual & yes \\
\hline & Matei et al. & crime & individual & yes \\
\hline 2003 & Weiner and Harris & land use & focus group & no \\
\hline 2005 & Doran and Lees & crime & individual & no \\
\hline 2006 & Pain et al. & crime & individual & no \\
\hline \multirow[t]{2}{*}{2007} & $\begin{array}{l}\text { Hall-Arber and St } \\
\text { Martin }\end{array}$ & U.S. & interview & yes \\
\hline & Dongus et al. & public health & focus group & no \\
\hline 2008 & D'Antona et al. & land use & household & no \\
\hline 2009 & $\begin{array}{l}\text { Brennan-Horley and } \\
\text { Gibson }\end{array}$ & creative industry & individual & yes \\
\hline \multirow[t]{6}{*}{2010} & $\begin{array}{l}\text { Fielding and Cisneros- } \\
\text { Puebla }\end{array}$ & public health & focus group & no \\
\hline & Beyer et al. & public health & $\begin{array}{l}\text { individual and } \\
\text { focus group }\end{array}$ & yes \\
\hline & Brennan-Horley et al. & creative industry & individual & yes \\
\hline & Lopez and Lukinbeal & crime & individual & yes \\
\hline & Sletto et al. & $\begin{array}{l}\text { environmental } \\
\text { hazards }\end{array}$ & focus group & no \\
\hline & Wridt & public health & focus group & yes \\
\hline \multirow[t]{2}{*}{2011} & Boschmann & employment & individual & yes \\
\hline & Doran and Burgess & crime & individual & yes \\
\hline \multirow[t]{2}{*}{2014} & $\begin{array}{l}\text { Boschmann and } \\
\text { Cubbon }\end{array}$ & review article & & \\
\hline & Curtis et al. & crime & individual & yes \\
\hline
\end{tabular}


2015 O'Neill et al.

Pearsall et al.

2016 Curtis

Manton et al.

Sloan et al.

2017 Hohenthal et al.

2018 Bereitschaft

Coquet et al.

Klonner et al.

Styers et al.

2019 Curtis et al. environmental individual yes hazards

higher education individual yes

review article

recreation individual yes

crime individual yes

public health focus group yes

creative industry individual yes

environmental individual yes

hazards

environmental individual yes

hazards

recreation individual yes

crime individual yes 
Table 2. Verbs for Digitizing Data with qualmap

\begin{tabular}{lll} 
Verb & Function & Definition \\
\hline define & qm_define () & Enter ID numbers for a single cluster \\
validate & qm_validate() & Check ID numbers to ensure they are valid \\
preview & qm_preview() & Plot cluster on an interactive map to visually confirm \\
create & qm_create() & Create cluster object with metadata \\
combine & qm_combine () & Combine multiple cluster objects together \\
bind & qm_bind () & Merge multiple objects created with qm_combine() \\
summarize & qm_summarize() & $\begin{array}{l}\text { Create counts of a single qualitative construct for } \\
\text { mapping }\end{array}$
\end{tabular}




\section{Figures}

Figure 1. Semi-structured Sketch Map Workflow

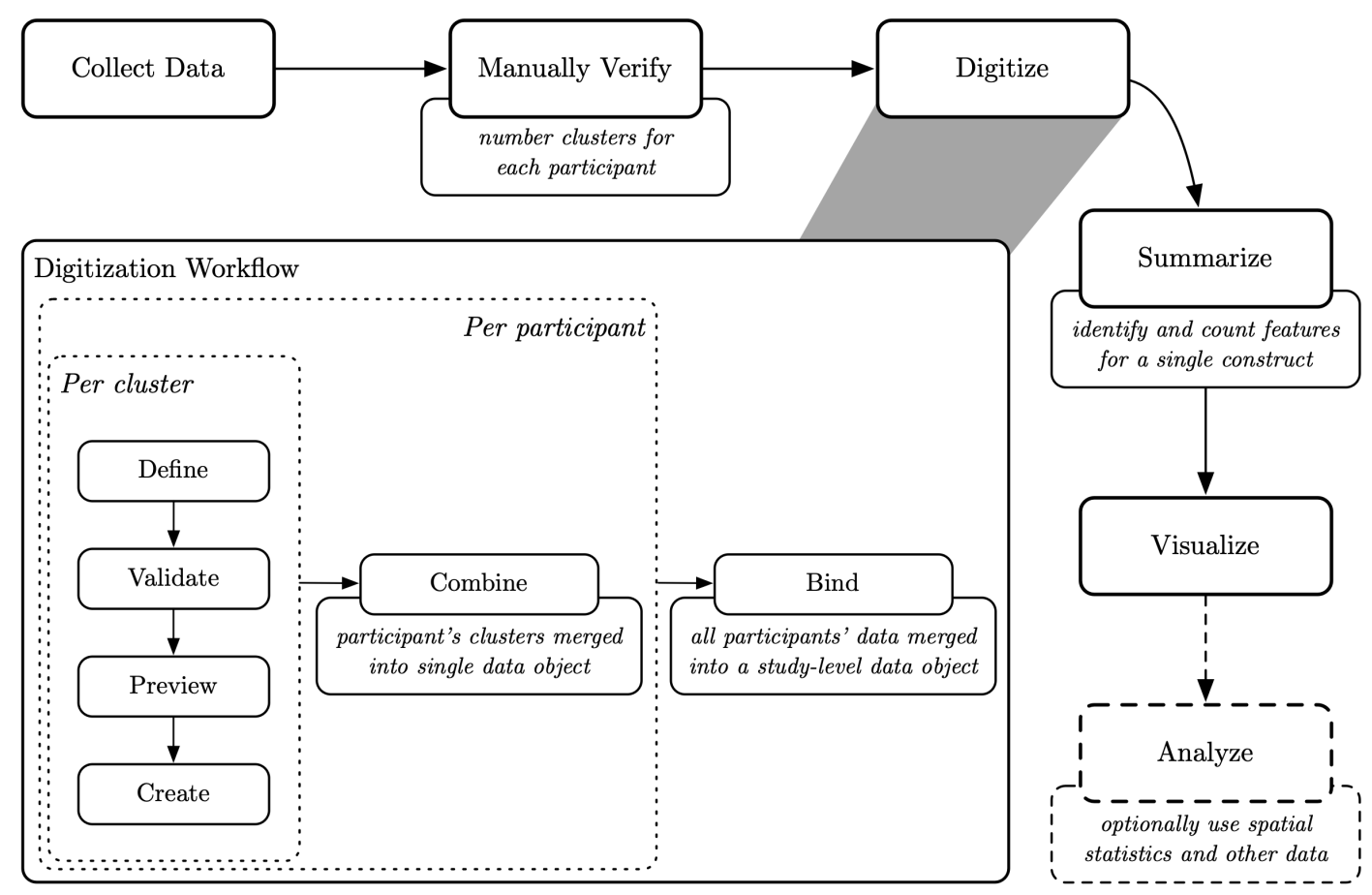


Figure 2. Example of labeled areal units for digitization as well as clusters with manual verification (numbering applied to each)

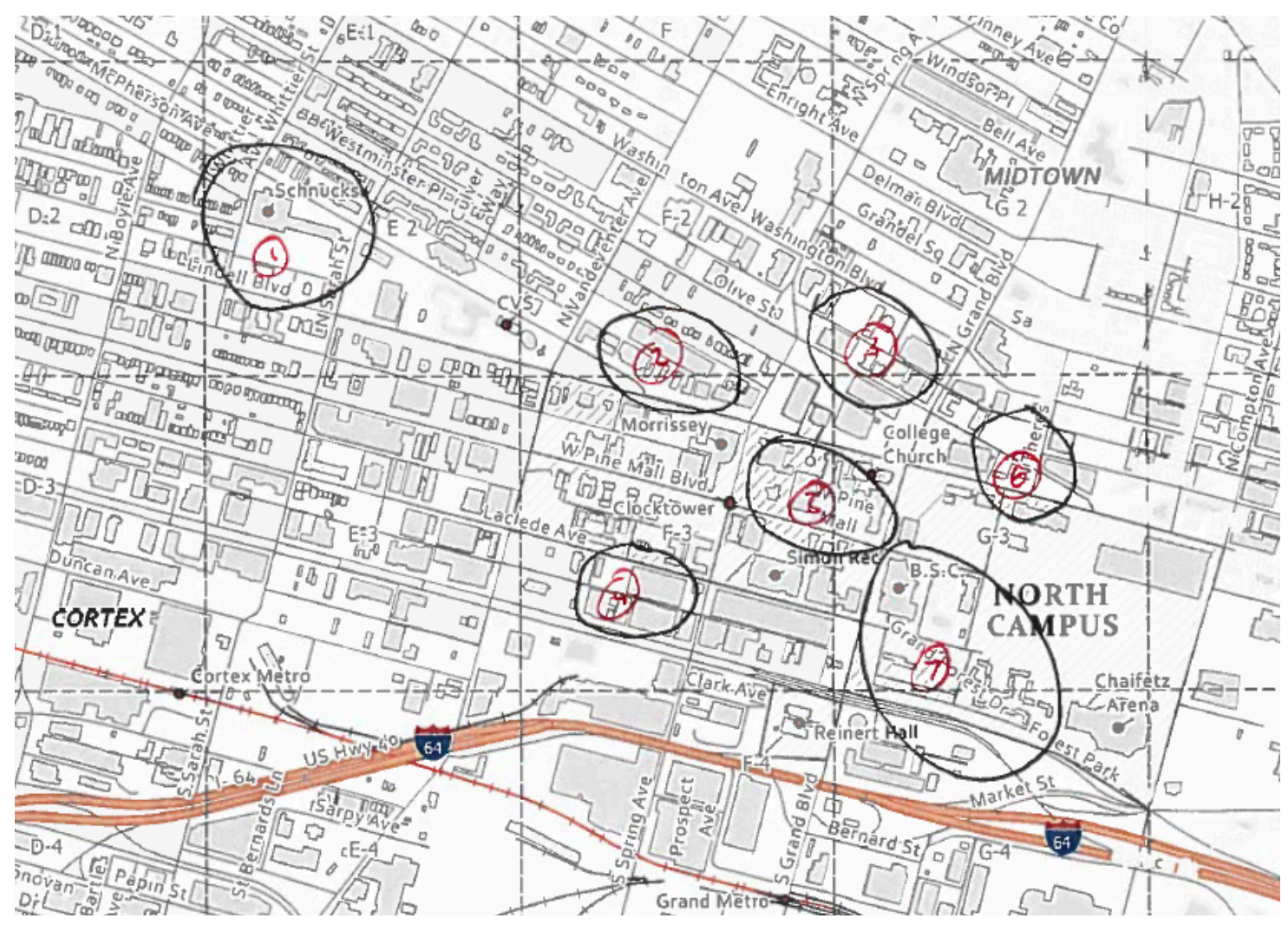


Figure 3. Frequent Visits (at least twice per week) by Respondents

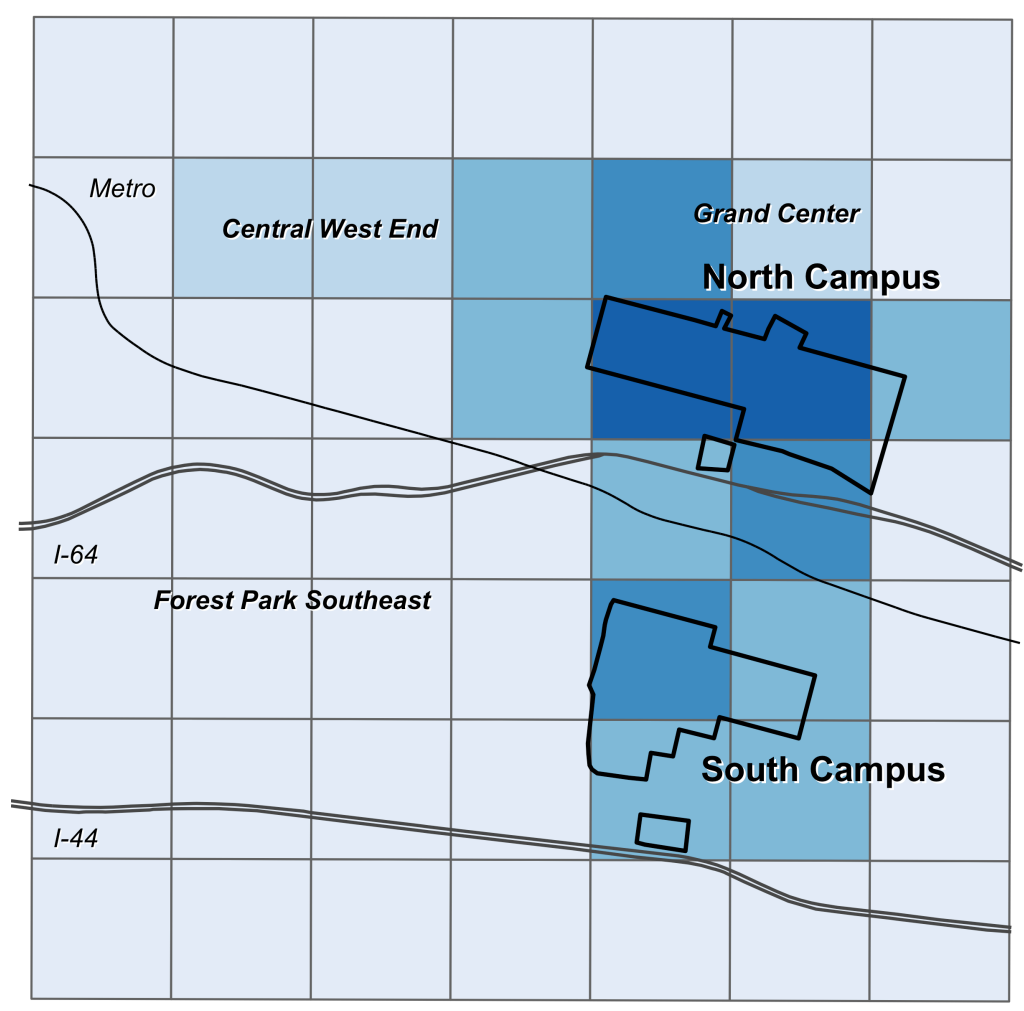

$\%$ of Respondents

$$
\begin{aligned}
& 0.0 \text { to } 8.0 \\
& 8.0 \text { to } 16.5 \\
& 16.5 \text { to } 33.0 \\
& 33.0 \text { to } 66.0 \\
& 66.0 \text { to } 100.0
\end{aligned}
$$

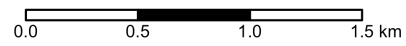


Figure 4. Regular Visits (at most three to four times per month) by Respondents

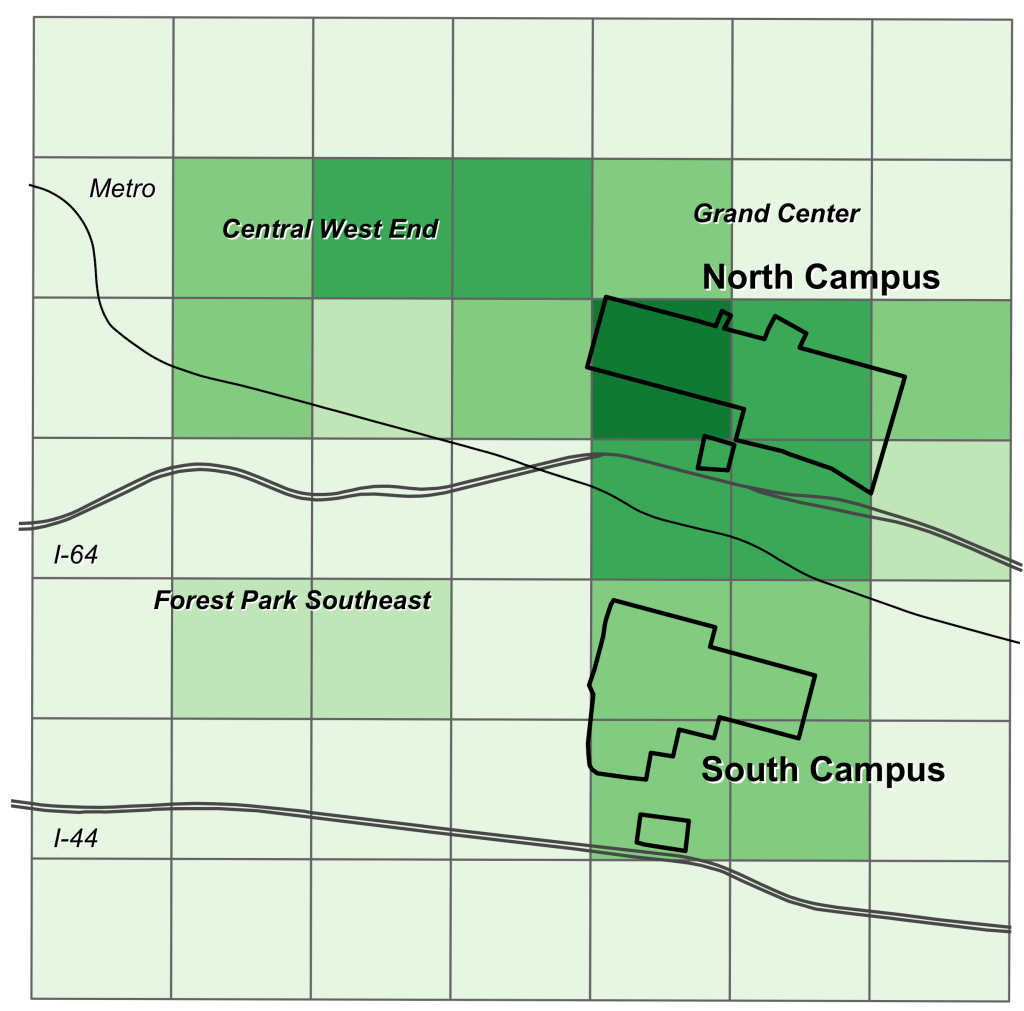

$\%$ of Respondents

0.0 to 8.0
8.0 to 16.5
16.5 to 33.0
33.0 to 49.5
49.5 to 66.0

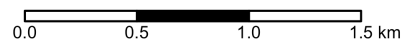


Figure 5. Frequent and Regular Visits Compared with Part 1 Crime Rates

A $\%$ of Respondents with Frequent Visits

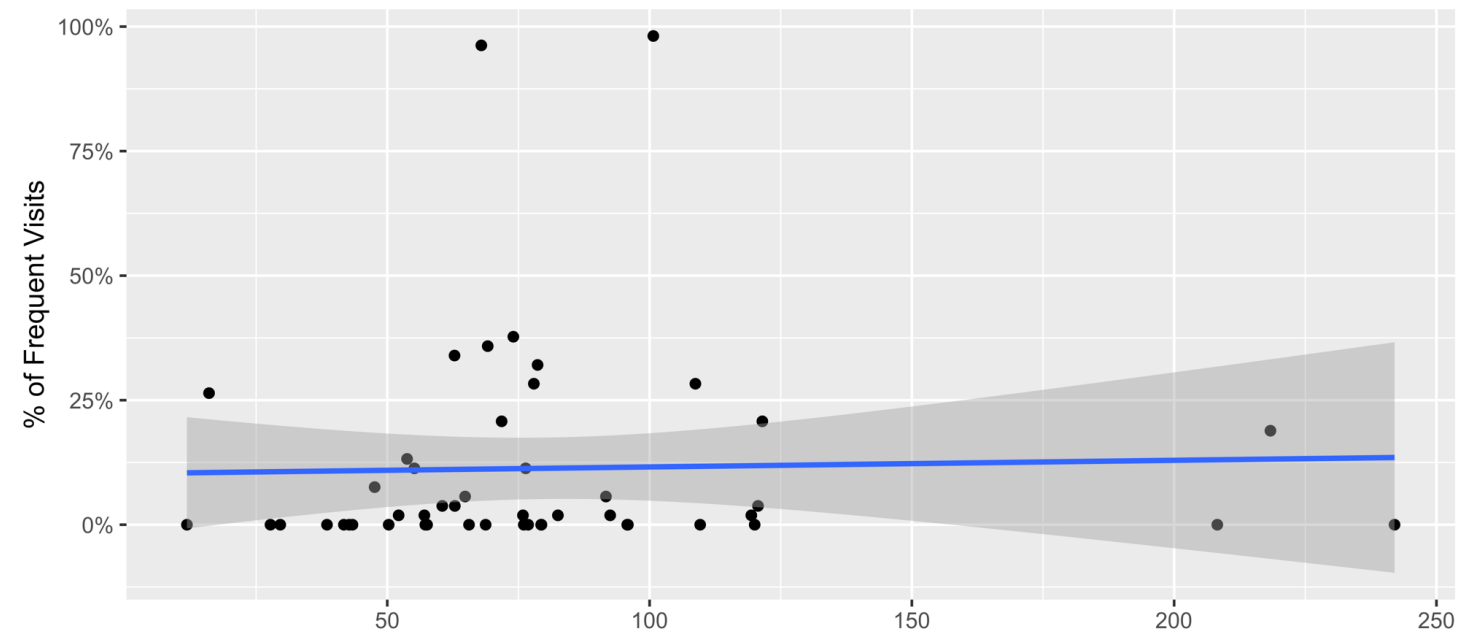

B $\%$ of Respondents with Regular Visits

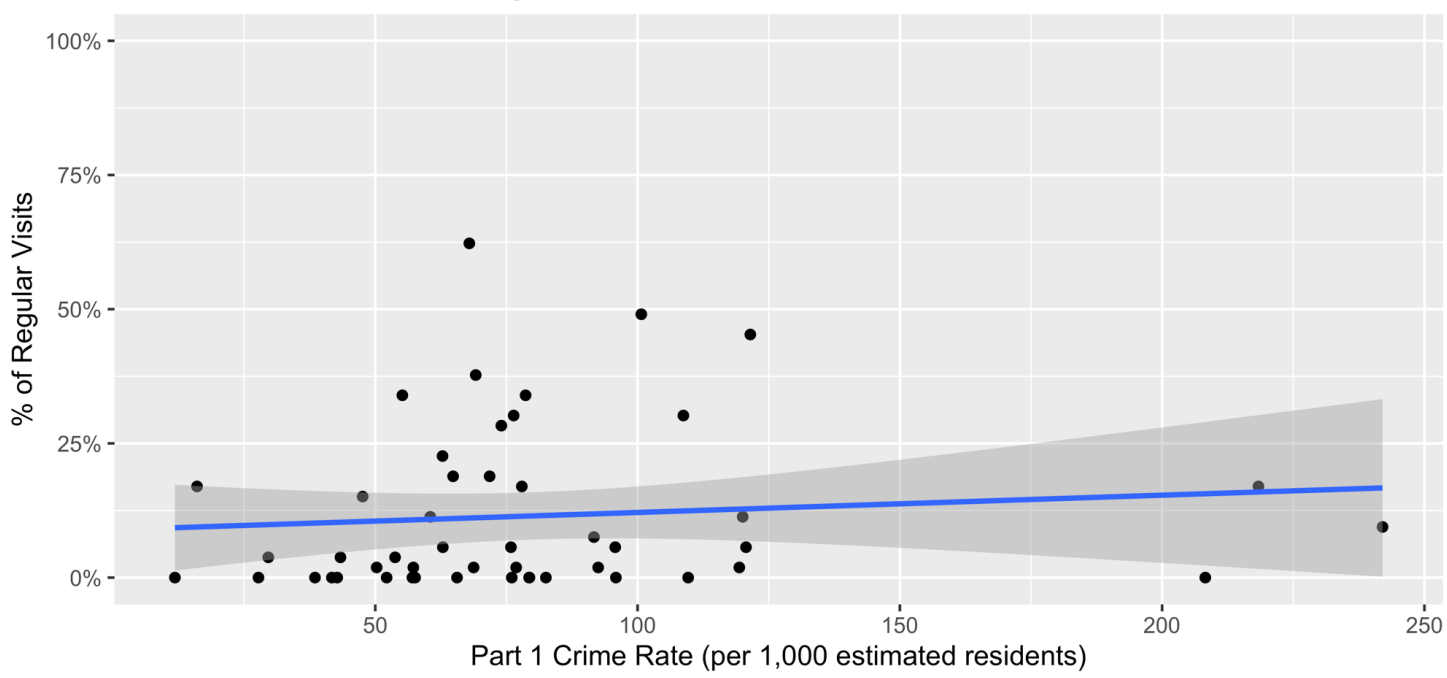




\section{End Notes}

[1] Geographers and others who utilize sketch mapping approaches have not fully embraced the distinction that Boschmann and Cubbon (2014) make between sketch mapping and mental mapping. Despite using cartographically accurate base maps and citing others who describe this as sketch mapping, some (e.g., Manton et al. 2016) use the term mental mapping. Though not all researchers make the distinction, it is clear that there are concrete differences between mental mapping in the vein of (Lynch 1960) and more recent approaches to sketch mapping, and this present paper will follow Boschmann and Cubbon (2014) in distinguishing between the two.

[2] Details on qualmap are available on the package's website $(<$ url redacted $>)$ as well as on its GitHub page $(<$ url redacted $>$ ). The software is available for download from both GitHub and the official R CRAN repository.

[3] A sample of the cover sheet and both map layouts are all available via the project's Open Science Framework page, available at $<$ url redacted for peer review $>$.

[4] As part of the author's commitment to reproducibility, all code and digitized data are available on this paper's GitHub page $(<$ url redacted $>$ ), and the corresponding sketch maps are cataloged on the project's Open Science Framework page $(<$ url redacted $>$ ). The final data set has also been made available within the qualmap package as sample data for users interested in the method to work with.

[5] The FBI defines eight significant categories of felony crimes as "Part 1" crimes: homicides, rapes, aggravated assaults, robberies, burglaries, larcenies, motor vehicle thefts, and arson fires. 


\section{Works Cited}

Agger, Ben. 1991. "Critical Theory, Poststructuralism, Postmodernism: Their Sociological Relevance." Annual Review of Sociology 17 (1): 105-31.

Andersson, Johan, Joanna Sadgrove, and Gill Valentine. 2012. "Consuming Campus: Geographies of Encounter at a British University." Social $\mathscr{E}$ Cultural Geography 13 (5): 501-15.

Bell, Scott, and Maureen Reed. 2006. "Adapting to the Machine: Integrating GIS into Qualitative Research." Cartographica: The International Journal for Geographic Information and Geovisualization, September.

Bereitschaft, Bradley. 2018. "Mapping Creative Spaces in Omaha, NE: Resident Perceptions Versus Creative Firm Locations." ISPRS International Journal of Geo-Information 7 (7): 263.

Beyer, Kirsten M. M., Sara Comstock, and Renea Seagren. 2010. "Disease Maps as Context for Community Mapping: A Methodological Approach for Linking Confidential Health Information with Local Geographical Knowledge for Community Health Research." Journal of Community Health 35 (6): 635-44.

Bivand, Roger S., Edzer Pebesma, and Virgilio Gomez-Rubio. 2013. Applied Spatial Data Analysis with R, Second Edition. Springer, NY.

Bivand, Roger, and David W. S. Wong. 2018. "Comparing Implementations of Global and Local Indicators of Spatial Association." TEST 27 (3): 716-48.

Boschmann, E. Eric. 2011. "Job Access, Location Decision, and the Working Poor: A Qualitative Study in the Columbus, Ohio Metropolitan Area." Geoforum 42 (6): $671-82$.

Boschmann, E. Eric, and Emily Cubbon. 2014. "Sketch Maps and Qualitative GIS: Using Cartographies of Individual Spatial Narratives in Geographic Research." The Professional Geographer 66 (2): 236-48.

Brennan-Horley, Chris, and Chris Gibson. 2009. "Where Is Creativity in the City? Integrating Qualitative and GIS Methods." Environment and Planning A: Economy and Space 41 (11): 2595-2614.

Brennan-Horley, Chris, Susan Luckman, Chris Gibson, and Julie Willoughby-Smith. 2010. "GIS, Ethnography, and Cultural Research: Putting Maps Back into Ethnographic Mapping." The Information Society 26 (2): 92-103.

Ceccato, Vania A, and Folke Snickars. 2000. "Adapting GIS Technology to the Needs of Local Planning." Environment and Planning B: Planning and Design 27 (6): 92337.

Cheng, Joe, Bhaskar Karambelkar, and Yihui Xie. 2019. Leaflet: Create Interactive Web Maps with the JavaScript 'Leaflet' Library.

Cieri, Marie. 2003. "Between Being and Looking Queer Tourism Promotion and Lesbian Social Space in Greater Philadelphia." ACME 2: 147-66.

Coquet, Marie, Denis Mercier, and Ghozlane Fleury-Bahi. 2018. "Individuals' Perceptions of Areas Exposed to Coastal Flooding in Four French Coastal 
Municipalities: The Contribution of Sketch Mapping." Geoenvironmental Disasters 5 (1): 15.

Coulton, Claudia J., Jill Korbin, Tsui Chan, and Marilyn Su. 2001. "Mapping Residents' Perceptions of Neighborhood Boundaries: A Methodological Note." American Journal of Community Psychology 29 (2): 371-83.

Creswell, John W, and J David Creswell. 2017. Research Design: Qualitative, Quantitative, and Mixed Methods Approaches. Sage publications.

Curtis, Andrew, Jacqueline W. Curtis, Jayakrishnan Ajayakumar, Eric Jefferis, and Susanne Mitchell. 2019. "Same Space - Different Perspectives: Comparative Analysis of Geographic Context Through Sketch Maps and Spatial Video Geonarratives." International Journal of Geographical Information Science 33 (6): $1224-50$.

Curtis, Jacqueline W. 2016. "Transcribing from the Mind to the Map: Tracing the Evolution of a Concept." Geographical Review 106 (3): 338-59.

Curtis, Jacqueline W, Ellen Shiau, Bryce Lowery, David Sloane, Karen Hennigan, and Andrew Curtis. 2014. "The Prospects and Problems of Integrating Sketch Maps with Geographic Information Systems to Understand Environmental Perception: A Case Study of Mapping Youth Fear in Los Angeles Gang Neighborhoods." Environment and Planning B: Planning and Design 41 (2): 251-71.

De Oliveira D'Antona, Alvaro, Anthony D. Cak, and Leah K. VanWey. 2008. "Collecting Sketch Maps to Understand Property Land Use and Land Cover in Large Surveys." Field Methods 20 (1): 66-84.

Dohan, Daniel, and Martín Sánchez-Jankowski. 1998. "Using Computers to Analyze Ethnographic Field Data: Theoretical and Practical Considerations." Annual Review of Sociology 24 (1): 477-98.

Dongus, Stefan, Dickson Nyika, Khadija Kannady, Deo Mtasiwa, Hassan Mshinda, Ulrike Fillinger, Axel W. Drescher, Marcel Tanner, Marcia C. Castro, and Gerry F. Killeen. 2007. "Participatory Mapping of Target Areas to Enable Operational Larval Source Management to Suppress Malaria Vector Mosquitoes in Dar Es Salaam, Tanzania." International Journal of Health Geographics 6 (1): 37.

Doran, Bruce J., and Melissa B. Burgess. 2011. Putting Fear of Crime on the Map: Investigating Perceptions of Crime Using Geographic Information Systems. Springer Science \& Business Media.

Doran, Bruce J., and Brian G. Lees. 2005. "Investigating the Spatiotemporal Links Between Disorder, Crime, and the Fear of Crime." The Professional Geographer $57(1): 1-12$.

Eccles, Stuart. 2015. "The Rise of Opinionated Software." Medium. https://medium.com/@stueccles/the-rise-of-opinionated-software-ca1ba0140d5b.

Elwood, Sarah, and Meghan Cope. 2009. "Introduction: Qualitative GIS: Forging Mixed Methods Through Representations, Analytical Innovations, and Conceptual Engagements." Qualitative GIS: A Mixed Methods Approach, 1-12. 
Fielding, Nigel, and César A. Cisneros-Puebla. 2010. "CAQDAS-GIS Convergence: Toward a New Integrated Mixed Method Research Practice?" Journal of Mixed Methods Research 3 (4): 349-70.

Gold, Christopher. 2016. "Tessellations in GIS: Part II-Making Changes." Geo-Spatial Information Science 19 (2): 157-67.

Goodchild, Michael. 2000. "The Current Status of GIS and Spatial Analysis." Journal of Geographical Systems 2 (1): 5-11.

Hall-Arber, Madeleine, and Kevin St Martin. 2007. "Environment and Development: (Re)connecting Community and Commons in New England Fisheries, USA." Participatory Action Research Approaches and Methods.

Hohenthal, Johanna, Paola Minoia, and Petri Pellikka. 2017. "Mapping Meaning: Critical Cartographies for Participatory Water Management in Taita Hills, Kenya." The Professional Geographer 69 (3): 383-95.

Holton, Mark. 2015. "I Already Know the City, I Don't Have to Explore It': Adjustments to 'Sense of Place' for 'Local' UK University Students." Population, Space and Place 21 (8): 820-31.

- 2016. "The Geographies of UK University Halls of Residence: Examining Students' Embodiment of Social Capital." Children's Geographies 14 (1): 63-76.

Holton, Mark, and Mark Riley. 2013. "Student Geographies: Exploring the Diverse Geographies of Students and Higher Education." Geography Compass 7 (1). Wiley Online Library: 61-74.

Hopkins, Peter. 2011. "Towards Critical Geographies of the University Campus: Understanding the Contested Experiences of Muslim Students." Transactions of the Institute of British Geographers 36 (1): 157-69.

Jerolmack, Colin, and Alexandra K. Murphy. 2017. "The Ethical Dilemmas and Social Scientific Trade-Offs of Masking in Ethnography." Sociological Methods \& Research, March, 0049124117701483.

Klonner, Carolin, Tomás J. Usón, Sabrina Marx, Franz-Benjamin Mocnik, and Bernhard Höfle. 2018. "Capturing Flood Risk Perception via Sketch Maps." ISPRS International Journal of Geo-Information 7 (9): 359.

Knigge, LaDona, and Meghan Cope. 2006. "Grounded Visualization: Integrating the Analysis of Qualitative and Quantitative Data Through Grounded Theory and Visualization." Environment and Planning A 38 (11): 2021-37.

Kwan, Mei-Po. 2002. "Feminist Visualization: Re-Envisioning GIS as a Method in Feminist Geographic Research." Annals of the Association of American Geographers 92 (4): 645-61.

Kwan, Mei-Po, and LaDona Knigge. 2006. "Doing Qualitative Research Using GIS: An Oxymoronic Endeavor?" Environment and Planning A 38: 1999-2002.

Lake, Robert W. 1993. "Planning and Applied Geography: Positivism, Ethics, and Geographic Information Systems." Progress in Human Geography 17 (3): 404-13. 
Lopez, Natalie, and Chris Lukinbeal. 2010. "Comparing Police and Residents' Perceptions of Crime in a Phoenix Neighborhood Using Mental Maps in GIS." Yearbook of the Association of Pacific Coast Geographers 72: 33-55.

Lynch, Kevin. 1960. The Image of the City. MIT Press.

Manton, Richard, Henrike Rau, Frances Fahy, Jerome Sheahan, and Eoghan Clifford. 2016. "Using Mental Mapping to Unpack Perceived Cycling Risk." Accident Analysis 83 Prevention 88 (March): 138-49.

Matei, Sorin, Sandra Ball-Rokeach, and Jack Qiu. 2001. "Fear and Misperception of Los Angeles Urban Space: A Spatial-Statistical Study of Communication-Shaped Mental Maps." Communication Research 28 (4): 429-63.

Matthews, Stephen A., James E. Detwiler, and Linda M. Burton. 2006. "GeoEthnography: Coupling Geographic Information Analysis Techniques with Ethnographic Methods in Urban Research." Cartographica: The International Journal for Geographic Information and Geovisualization, October.

Morse, Janice M. 2016. Mixed Method Design : Principles and Procedures. Routledge. O'Neill, Eoin, Michael Brennan, Finbarr Brereton, and Harutyun Shahumyan. 2015. "Exploring a Spatial Statistical Approach to Quantify Flood Risk Perception Using Cognitive Maps." Natural Hazards 76 (3): 1573-1601.

Pain, Rachel, Robert MacFarlane, Keith Turner, and Sally Gill. 2006. "When, Where, If, and but': Qualifying GIS and the Effect of Streetlighting on Crime and Fear." Environment and Planning A: Economy and Space 38 (11): 2055-74.

Parker, Hilary. 2017. "Opinionated Analysis Development." PeerJ Preprints 5: e3210v1. Pavlovskaya, Marianna. 2006. "Theorizing with GIS: A Tool for Critical Geographies?" Environment and Planning A: Economy and Space 38 (11): 2003-20.

—. 2009. "Non-Quantitative GIS." Qualitative GIS: A Mixed Methods Approach, 13-37.

Pavlovskaya, Marianna E. 2002. "Mapping Urban Change and Changing GIS: Other Views of Economic Restructuring." Gender, Place and Culture: A Journal of Feminist Geography 9 (3): 281-89.

Pearsall, Hamil, Timothy Hawthorne, Daniel Block, Barbara Louise Endemaño Walker, and Michele Masucci. 2015. "Exploring Youth Socio-Spatial Perceptions of Higher Education Landscapes Through Sketch Maps." Journal of Geography in Higher Education 39 (1): 111-30.

Pebesma, Edzer. 2018. "Simple Features for R: Standardized Support for Spatial Vector Data." The R Journal 10 (1): 439-46.

Pebesma, Edzer J., and Roger S. Bivand. 2005. "Classes and Methods for Spatial Data in R." R News 5 (2): 9-13.

Pickles, John. 1995. "Representations in an Electronic Age: Geography, GIS, and Democracy." In Ground Truth: The Social Implications of Geographic Information Systems, edited by John Pickles, 1-30. New York, NY: The Guilford Press. 
Prener, Christopher G. 2020. "Finding the City in Sociology: Broadening and Deepening the Geographic Scope of the Urban and Inequality Literatures." Sociology Compass 14 (2): e12756.

Prener, Christopher, and Charles Revord. 2019. "Areal: An R Package for Areal Weighted Interpolation." Journal of Open Source Software 4 (37): 1221.

R Core Team. 2019. R: A Language and Environment for Statistical Computing. Vienna, Austria: R Foundation for Statistical Computing.

Rey, Sergio J., and Luc Anselin. 2010. "PySAL: A Python Library of Spatial Analytical Methods." In Handbook of Applied Spatial Analysis: Software Tools, Methods and Applications, edited by Manfred M. Fischer and Arthur Getis, 175-93. Berlin, Heidelberg: Springer.

Sandoval, J. S. Onesimo. 2013. "Understanding the Demographic Hurdles to Revitalize Saint Louis." Saint Louis University Public Law Review 33: 161-82.

Sletto, Bjørn. 2015. "Inclusions, Erasures and Emergences in an Indigenous Landscape: Participatory Cartographies and the Makings of Affective Place in the Sierra de Perijá, Venezuela." Environment and Planning D: Society and Space 33 (5): 92544.

Sletto, Bjørn, Solange Muñoz, Shawn M. Strange, Rosa E. Donoso, and Martin Thomen. 2010. "El Rincón de Los Olvidados": Participatory GIS, Experiential Learning and Critical Pedagogy in Santo Domingo, Dominican Republic." Journal of Latin American Geography 9 (3): 111-35.

Sloan, Nicola, Bruce Doran, Francis Markham, and Kristen Pammer. 2016. "Does Base Map Size and Imagery Matter in Sketch Mapping?" Applied Geography 71 (June): 24-31.

Smith, Neil. 1979. "Geography, Science and Post-Positivist Modes of Explanation." Progress in Geography 3 (3): 356-83.

Smith, Tara A., and J. S. Onésimo Sandoval. 2019a. "Examining the Local Spatial Variability of Robberies in Saint Louis Using a Multi-Scale Methodology." Social Sciences 8 (2): 50.

- 2019b. "A Spatial Analysis of Homicides in Saint Louis: The Importance of Scale." Spatial Demography 7 (1): 57-82.

Styers, Diane M., G. Rebecca Dobbs, Lee K. Cerveny, and Isaac T. Hayes. 2018. "Geovisualization of Socio-Spatial Data on Outdoor Activities and Values in the Southern Appalachians." International Journal of Applied Geospatial Research (IJAGR) 9 (3): 55-80.

Teddlie, Charles, and Abbas Tashakkori. 2011. "Mixed Methods Research." The Sage Handbook of Qualitative Research 4: 285-300.

Tennekes, Martijn. 2018. "Tmap: Thematic Maps in R." Journal of Statistical Software $84(6): 1-39$.

Tuan, Yi-fu. 1975. "Images and Mental Maps." Annals of the Association of American Geographers 65 (2): 205-12. 
Wachnik, Klaudia. 2019. "SLU 'Safety Bubble': A Matter of Perception." The University News, September. https://unewsonline.com/2019/09/slu-safety-bubbleeffectiveness-questioned/.

Walker, Kyle. 2019a. Tidycensus: Load US Census Boundary and Attribute Data as 'Tidyverse' and 'Sf'-Ready Data Frames.

- 2019b. Tigris: Load Census TIGER/Line Shapefiles.

Weiner, Dan, and Trevor Harris. 2003. "Community-Integrated GIS for Land Reform." URISA Journal 15 (2).

Wickham, Hadley. 2016. Ggplot2: Elegant Graphics for Data Analysis. Springer-Verlag New York.

Wickham, Hadley, Mara Averick, Jennifer Bryan, Winston Chang, Lucy McGowan, Romain François, Garrett Grolemund, et al. 2019. "Welcome to the Tidyverse." Journal of Open Source Software 4 (43): 1686.

Wilke, Claus O. 2019. Cowplot: Streamlined Plot Theme and Plot Annotations for 'Ggplot2'.

Wridt, Pamela. 2010. "A Qualitative GIS Approach to Mapping Urban Neighborhoods with Children to Promote Physical Activity and Child-Friendly Community Planning." Environment and Planning B: Planning and Design 37 (1): 129-47.

Yu, Shaolu, Madalyn Bryant, Emily Messmer, Sophia Tsagronis, and Sarah Link. 2018. "'Is There a Bubble to Burst?'-College Students' Spatial Perception of Campus and the City, a Case Study of Rhodes College in Memphis, TN." Urban Geography 39 (10): 1555-75. 